\title{
Physics in Space beyond the Schwarzschild Radius
}

\author{
Jaroslav Hynecek ${ }^{1}$ \\ ${ }^{1}$ Isetex, Inc., 905 Pampa Drive, Allen, TX 75013, USA \\ Correspondence: Jaroslav Hynecek, Isetex, Inc., 905 Pampa Drive, Allen, TX 75013, USA. E-mail: \\ jhynecek@netscape.net
}

Received: March 27, 2013 Accepted: June 20, 2013 Online Published: July 9, 2013

doi:10.5539/apr.v5n4p46

URL: http://dx.doi.org/10.5539/apr.v5n4p46

\begin{abstract}
This paper investigates the spherical region of space that is bounded by the Schwarzschild radius. This is possible because the metric that describes this space-time is not the Schwarzschild metric and, therefore, does not have the Event Horizon and Black Hole pathologies. It is found that in the certain finite region of this space-time the time, as observed by a distant observer, slows down so much that it is possible to explain charge of an electron as frozen vibrations of electromagnetic field. The same effect may also explain the long neutron lifetime, the stability of typical nuclei, and the variations of radioactive nuclei decay rates that correlate with the Earth-Sun distance.
\end{abstract}

Keywords: Schwarzschild radius, new MTG metric, Black Holes, Event Horizon, Christoffel coefficients, Riemann tensor, Ricci tensor, Ricci scalar, Lagrange formalism, neutron lifetime, model of electron charge, variation of nuclei decay rates

\section{Introduction}

The calculations presented in this paper are based on the new metric that was derived previously in the metric theory of gravity (MTG) and was published already several times with the latest version available in this journal (Hynecek, 2012). The metric describes the space-time without Black Holes (BH) and the Event Horizon. The correctness of the metric was verified, for example, by deriving formulas for the geodetic precession and comparing predictions with the measurement results of Gravity Probe B (Hynecek, 2013). Additional verification was made by investigating the famous light bending effect caused by a gravitating body (Hynecek, 2011). The author is thus certain that this metric describes the reality correctly and is therefore suitable for studying the Schwarzschild region of space-time. The Schwarzschild metric, however, with its typical BH region unfortunately does not permit to study anything in that region, because no data can be retrieved from it. From the positivistic philosophy point of view this region actually does not exist as a typical space-time as we know it. Whatever has been previously published about this region are sheer speculations and a belief that does not belong to the scientific world. The new metric, on the other hand, does not have such a problem, and thus it is possible to study the Schwarzschild region without any difficulty and find interesting properties of matter that originate in this space-time. This opens up a new frontier for study of gravity and perhaps also for the study that may be related to quantum physics in curved space-times (Birrell \& Davies, 1982). The quantum aspects of the theory, however, are not addressed in this paper and are deferred to future work.

\section{The New MTG Space-Time Metric, Christoffel Coefficients, and the Riemann and Ricci Tensors}

The differential metric line element for the new MTG metric is defined as:

$$
d s^{2}=g_{t t}(c d t)^{2}-d \rho^{2}-g_{t t} \rho^{2} d \Omega^{2}
$$

where for the usual variables holds the following: $d \rho=g_{t t}^{-1 / 2} d r, g_{t t}=\exp \left(-R_{s} / \rho\right), d \Omega^{2}=d \vartheta^{2}+\sin ^{2} \vartheta d \varphi^{2}$, and where the Schwarzschild radius is defined as: $R_{s}=2 \kappa M / c^{2}$. In this metric and throughout this paper it is necessary to keep in mind that there are two types of coordinates used. The natural coordinates with the radial coordinate designated by $r$, and the physical radial coordinate designated by $\rho$. The physical coordinates are not directly observable the same way as the natural coordinates are and must therefore be calculated from some applicable formulas. This also applies to the physical radius, which is calculated from the formula: 


$$
d \rho=\exp \left(R_{s} / 2 \rho\right) d r
$$

The solution of this differential equation cannot be found in a closed analytical form and needs to be calculated numerically. The graph of this solution is shown in Figure 1, where both variables were normalized to the Schwarzschild radius. From the graph it can be clearly observed that the natural radius is significantly compressed in the region below the Schwarzschild radius bound.

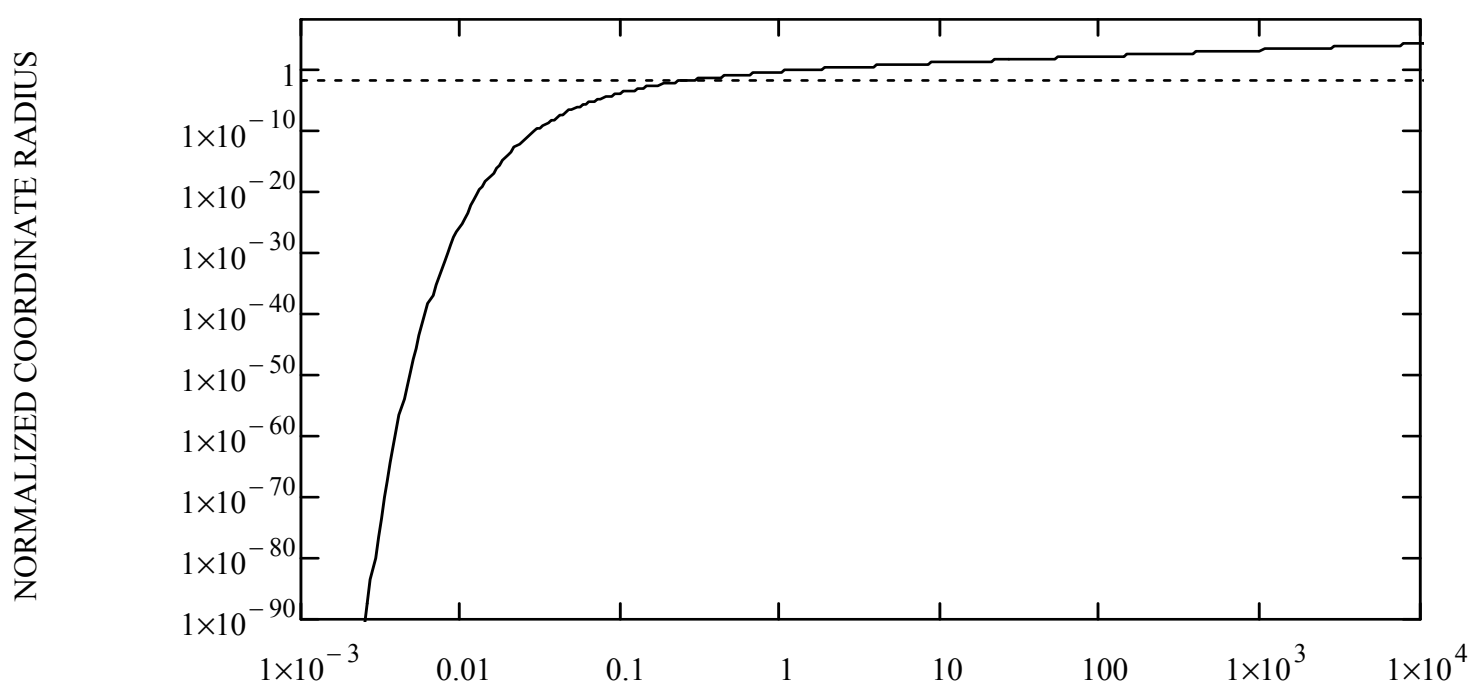

NORMALIZED PHYSICAL RADIUS

Figure 1. Normalized natural radius as a function of the normalized physical radius. The dotted line represents the natural mass equivalent radius corresponding to the equilibrium between the integrated field energy in the space outside of this radius and the mass equivalent energy of the gravitating body

The dotted line designates the natural mass equivalent radius $r_{\mathrm{e}}$ which corresponds to the minimum radius to which any ordinary mass can be compacted to and at the same time satisfy the mass energy equivalence condition. This means that the field energy of the gravitating body integrated from this radius to infinity is equal to the equivalent mass energy of the body according to the formula:

$$
M c^{2}=\frac{1}{2} \frac{\kappa M^{2}}{\rho_{e}\left(r_{e}\right)}
$$

In this formula $\kappa$ designates the Newton gravitational constant and $M$ the mass of the centrally gravitating body. This condition, however, does not state how the mass of the compacted body is organized and structured in the region bounded by $\rho_{e}$.

To complete the description of space-time, the Christoffel coefficients, the Riemann and Ricci tensors, and the Ricci scalar for the new MTG metric are introduced below as they were previously computed and published (Hynecek, 2007). The metric signature notation is sometimes changed for a convenience from $(t,-r,-\theta,-\varphi)$ to $(0,-1,-2,-3)$, but this should not present a problem. The Christoffel coefficients are thus as follows:

$$
\begin{aligned}
& \Gamma_{01}^{0}=\frac{1}{\rho^{2}} \frac{R_{s}}{2} e^{\frac{1 R_{s}}{2 \rho}} \\
& \Gamma_{00}^{1}=\frac{1}{\rho^{2}} \frac{R_{s}}{2} e^{\frac{-3 R_{s}}{2}} \quad \Gamma_{11}^{1}=-\frac{1}{\rho^{2}} \frac{R_{s}}{2} e^{\frac{1 R_{s}}{2 \rho}} \quad \Gamma_{22}^{1}=-\left(\rho+\frac{R_{s}}{2}\right) e^{\frac{-3}{2} \rho R_{s}} \quad \Gamma_{33}^{1}=\Gamma_{22}^{1} \sin ^{2} \vartheta \\
& \Gamma_{12}^{2}=\frac{1}{\rho^{2}}\left(\rho+\frac{R_{s}}{2}\right) e^{\frac{1}{2} \frac{R_{s}}{\rho}} \quad \Gamma_{33}^{2}=-\sin \vartheta \cos \vartheta
\end{aligned}
$$




$$
\Gamma_{13}^{3}=\frac{1}{\rho^{2}}\left(\rho+\frac{R_{s}}{2}\right) e^{\frac{1}{2} \frac{R_{s}}{\rho}} \quad \Gamma_{23}^{3}=\frac{\cos \vartheta}{\sin \vartheta}
$$

The Riemann tensor is calculated according to the relation:

$$
R_{\mu \nu \sigma}^{\lambda}=\partial_{\nu} \Gamma_{\mu \sigma}^{\lambda}-\partial_{\sigma} \Gamma_{\mu \nu}^{\lambda}+\Gamma_{\mu \sigma}^{\eta} \Gamma_{\eta v}^{\lambda}-\Gamma_{\mu \nu}^{\eta} \Gamma_{\eta \sigma}^{\lambda}
$$

with the following results:

$$
\begin{array}{ll}
R_{101}^{0}=\frac{1}{\rho^{4}} \frac{R_{s}}{2}\left(2 \rho-\frac{R_{s}}{2}\right) e^{\frac{R_{s}}{\rho}} & R_{020}^{2}=\frac{1}{\rho^{4}} \frac{R_{s}}{2}\left(\rho+\frac{R_{s}}{2}\right) e^{\frac{-R_{s}}{\rho}} \\
R_{202}^{0}=-\frac{1}{\rho^{2}} \frac{R_{s}}{2}\left(\rho+\frac{R_{s}}{2}\right) e^{\frac{-R_{s}}{\rho}} & R_{121}^{2}=-\frac{1}{\rho^{4}}\left(\frac{R_{s}}{2}\right)^{2} e^{\frac{R_{s}}{\rho}} \\
R_{303}^{0}=-\frac{1}{\rho^{2}} \frac{R_{s}}{2}\left(\rho+\frac{R_{s}}{2}\right) e^{\frac{-R_{s}}{\rho}} \sin ^{2} \vartheta & R_{323}^{2}=\left[1-\frac{1}{\rho^{2}}\left(\rho+\frac{R_{s}}{2}\right)^{2} e^{\frac{-R_{s}}{\rho}}\right] \sin ^{2} \vartheta \\
R_{010}^{1}=-\frac{1}{\rho^{4}} \frac{R_{s}}{2}\left(2 \rho-\frac{R_{s}}{2}\right) e^{\frac{-R_{s}}{\rho}} & R_{030}^{3}=\frac{1}{\rho^{4}} \frac{R_{s}}{2}\left(\rho+\frac{R_{s}}{2}\right)^{\frac{-R_{s}}{\rho}} \\
R_{212}^{1}=-\frac{1}{\rho^{2}}\left(\frac{R_{s}}{2}\right)^{2} e^{\frac{-R_{s}}{\rho}} & R_{131}^{3}=-\frac{1}{\rho^{4}}\left(\frac{R_{s}}{2}\right)^{2} e^{\frac{R_{s}}{\rho}} \\
R_{313}^{1}=-\frac{1}{\rho^{2}}\left(\frac{R_{s}}{2}\right)^{2} e^{\frac{-R_{s}}{\rho}} \sin ^{2} \vartheta & R_{232}^{3}=1-\frac{1}{\rho^{2}}\left(\rho+\frac{R_{s}}{2}\right)^{2} e^{\frac{-R_{s}}{\rho}}
\end{array}
$$

where only the nonzero symmetric terms were listed. After contracting the Riemann tensor the Ricci tensor becomes:

$$
\begin{array}{ll}
R_{00}=\frac{3}{\rho^{4}}\left(\frac{R_{s}}{2}\right)^{2} e^{\frac{-R_{s}}{\rho}} & R_{0}^{0}=\frac{3}{\rho^{4}}\left(\frac{R_{s}}{2}\right)^{2} \\
R_{11}=\frac{1}{\rho^{4}} \frac{R_{s}}{2}\left(2 \rho-3 \frac{R_{s}}{2}\right) e^{\frac{R_{s}}{\rho}} & R_{1}^{1}=\frac{1}{\rho^{4}} \frac{R_{s}}{2}\left(-2 \rho+3 \frac{R_{s}}{2}\right) \\
R_{22}=1-\frac{1}{\rho^{2}}\left(\rho^{2}+3 \rho \frac{R_{s}}{2}+3\left(\frac{R_{s}}{2}\right)^{2}\right) e^{\frac{-R_{s}}{\rho}} & R_{2}^{2}=\frac{1}{\rho^{2}}\left(1-e^{\frac{R_{s}}{\rho}}\right)+\frac{3}{\rho^{4}} \frac{R_{s}}{2}\left(\rho+\frac{R_{s}}{2}\right) \\
R_{33}=R_{22} \sin ^{2} \vartheta & R_{3}^{3}=R_{2}^{2}
\end{array}
$$

The Ricci scalar then becomes as follows: 


$$
R_{c}=\frac{2}{\rho^{2}}\left(1-e^{\frac{R_{s}}{\rho}}\right)+\frac{2 R_{s}}{\rho^{3}}+\frac{3 R_{s}^{2}}{\rho^{4}}
$$

For small $R_{\mathrm{s}} / \rho$ this expression reduces to:

$$
R_{c} \approx \frac{2 R_{s}^{2}}{\rho^{4}} \cdots
$$

Having thus obtained the Ricci scalar for the new metric it is now possible to plot it as a function of the natural radial coordinate. This is shown in Figure 2 normalized to the Schwarzschild radius. It is interesting to observe that the Ricci scalar is very close to zero for the region where the natural radius is larger than the Schwarzschild radius, rises to a significantly large positive value for the radius in the interval: $0.03<r<1$, and then assumes very large negative values for the rest of the natural Schwarzschild region interval. This suggests a possibility for the existence of complicated and rich geometrical structures that may be found in this region of space. The near zero value of Ricci scalar outside of the Schwarzschild region is consistent with the assumption of Einstein's general relativity theory.

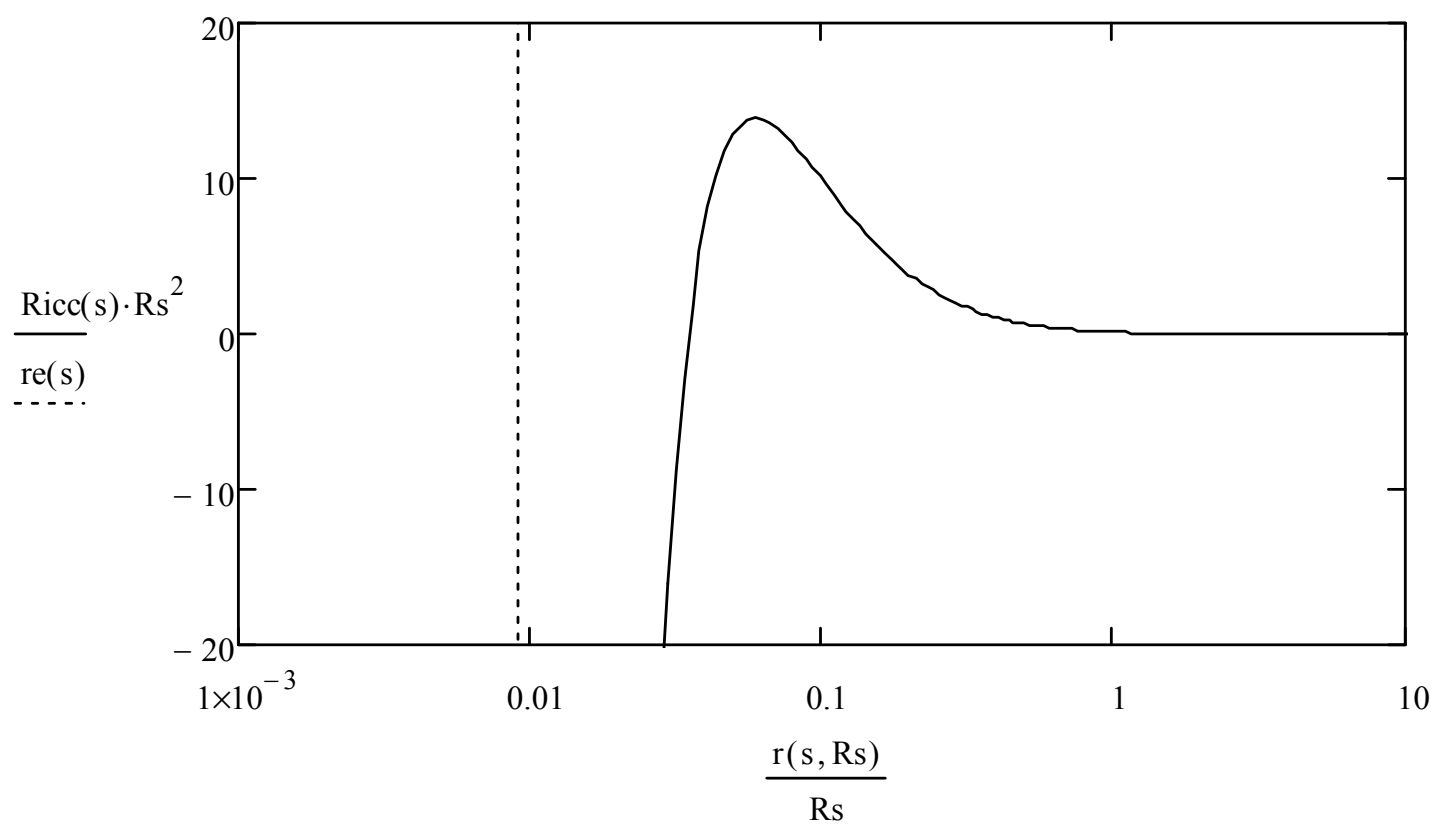

Figure 2. Normalized Ricci curvature as a function of the normalized natural radius $r(s)$ for a centrally gravitating body. The vertical dotted line indicates the location of the normalized mass equivalent natural radius

\section{Trajectories of Test Bodies in the Schwarzschild Region}

The first trajectory that will be investigates is the trajectory of a small test body falling in the radial direction. The formalism that will be used is the well-known and ages tested Lagrange formalism with the Lagrangian $\left(L=c^{2}\right)$ derived from the metric as follows:

$$
L=g_{t t}\left(\frac{c d t}{d \tau}\right)^{2}-\left(\frac{d \rho}{d \tau}\right)^{2}
$$

The first integrals of Euler-Lagrange equations that follow from the corresponding variational principle are:

$$
d \tau=g_{t t} d t
$$

$$
\left(\frac{d \rho}{d \tau}\right)^{2}=c^{2} e^{\frac{R_{s}}{\rho}}-c^{2}
$$


Using Equation 11 and Equation 12 it is possible to eliminate the physical variables from Equation 12 and obtain the formula for the velocity as observed by a distant observer:

$$
\frac{d r}{d t}= \pm c e^{\frac{-3 R_{s}}{2 \rho}} \sqrt{e^{\frac{R_{s}}{\rho}}-1}
$$

The two velocity solutions correspond to the in-falling and outgoing test body trajectories. It is also not too difficult to find the acceleration as observed by a distant observer. This is obtained by differentiating Equation 12 with respect to $\tau$ and again using Equation 11 and Equation 12 to eliminate the physical variables from the differentials. The result is as follows:

$$
\frac{d^{2} r}{d t^{2}}=-\frac{c^{2}}{2} \frac{R_{s}}{\rho^{2}}\left(3 e^{\frac{-5 R_{s}}{2 \rho}}-2 e^{\frac{-3 R_{s}}{2 \rho}}\right)
$$

The graph of velocities and the acceleration as functions of the normalized natural radius are shown in Figure 3.

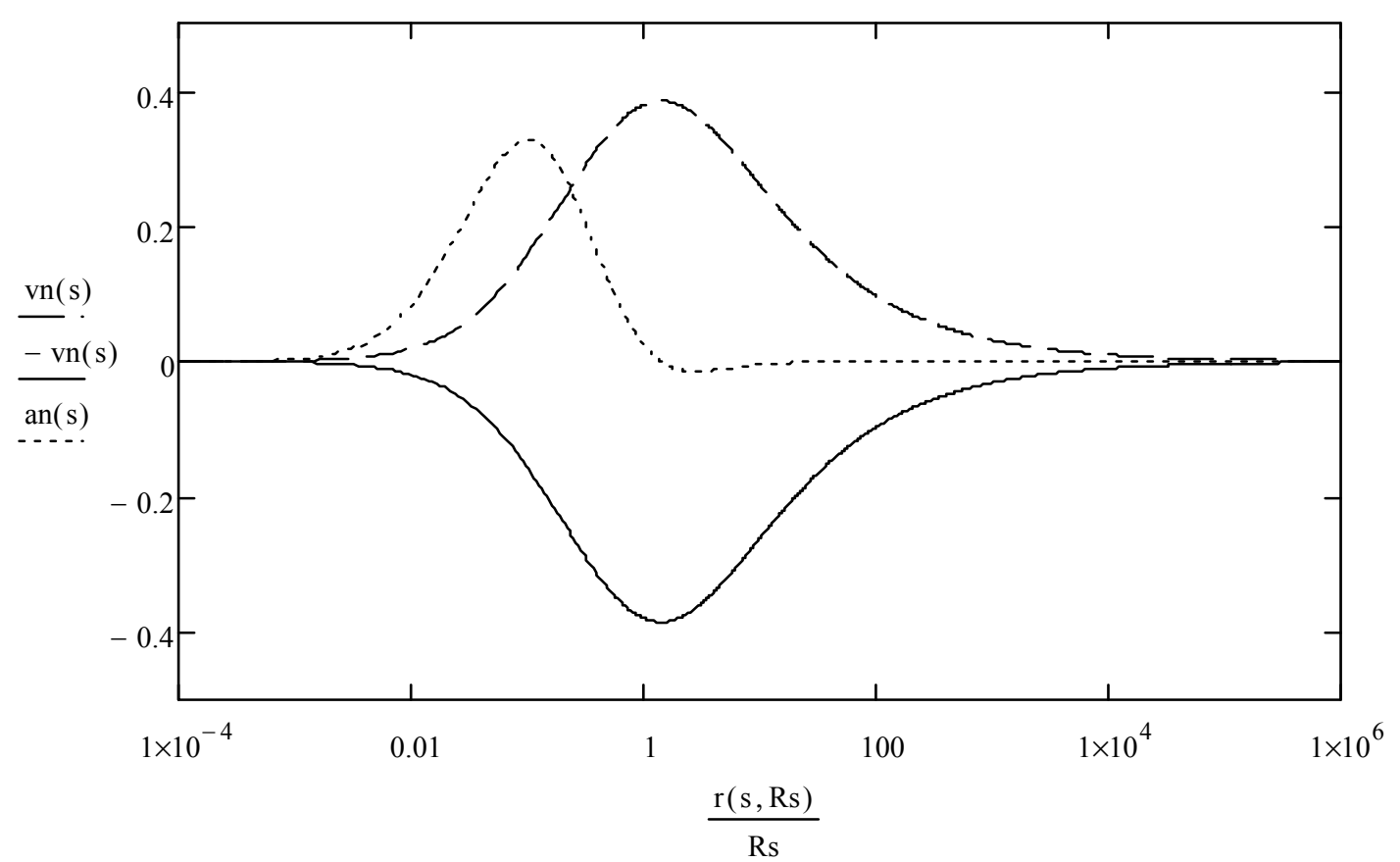

Figure 3. Dependencies of normalized velocities and the acceleration on normalized natural radius. The solid line is the velocity of in-falling particle, the dashed line is for the outgoing particle, and the dotted line is the acceleration

The remarkable observation that can be made in this graph is the very strong apparent deceleration for the incoming test particle in the region of: $0.01<r / R_{s}<1$, while the standard gravitational acceleration in the region: $r / R_{s}>1$, is relatively small in comparison and, of course, negative. This is astonishing and an unexpected finding for this region of space-time. It is also worth noting that for approximately: $r / R_{s}<0.001$, the acceleration is approaching zero, which implies that there is no apparent acting force. This resembles the asymptotic freedom postulated for quarks.

Another interesting point to note is that the velocity maximum occurs just before the Schwarzschild radius is reached and is equal to: $v_{\max }=0.385 \mathrm{c}$. This value is identical with the maximum galaxy recession velocity that can be observed from Earth as described in the Repulsive Dark Matter (RDM) model of the universe (Hynecek, 2012b). It thus seems that the physics of the universe and the physics of the Schwarzschild region share some common interesting features.

The graph in Figure 3 can also be used to explain other interesting observations, which are the particle jets (NASA, 2013) that emanate from the centers of many galaxies. Assuming that these centers are already compacted to their 
minimum sizes, corresponding to the mass equivalent natural radius of approximately: $r / R_{s}=0.01$, it can be observed that at that radius the in-falling test mass velocity is still considerable. This will result in some energy and momentum exchanges between the main body and the in-falling mass and perhaps ejection of new particles along the outgoing velocity curve (dashed line) just to conserve the linear momentum as in any similar collisions of small test bodies with a large mass. Of course, the details of this process are much more complicated, including the main body rotational effects and thus most likely its considerably non-spherical shape. However, the essential reasons that underline this phenomenon are definitely guided by the space-time geometry that exists in this Schwarzschild region.

In the next step of the study the trajectory of an orbiting test body will be investigated. For simplicity only the circular orbit will be analyzed. Such an orbit is again described by the following Lagrangian:

$$
L=g_{t t}\left(\frac{c d t}{d \tau}\right)^{2}-\left(\frac{d \rho}{d \tau}\right)^{2}-\rho^{2} g_{t t}\left(\frac{d \varphi}{d \tau}\right)^{2}
$$

Solving the corresponding Euler-Lagrange equations the formula for the orbital frequency as a function of radius is derived as was published by Hynecek (2010).

$$
\frac{d \varphi}{d t}=c \sqrt{\frac{\dot{g}_{t t}}{\dot{g}_{\varphi \varphi}}}=\frac{c}{\rho} \sqrt{\frac{R_{s}}{2 \rho+R_{s}}}
$$

The dot in Equation 16 represents the derivative with respect to $\rho$. The graph of the resulting dependency on the natural radius $r$ is shown in Figure 4 and it is referenced to the Schwarzschild radius value: $R_{s}=1$.

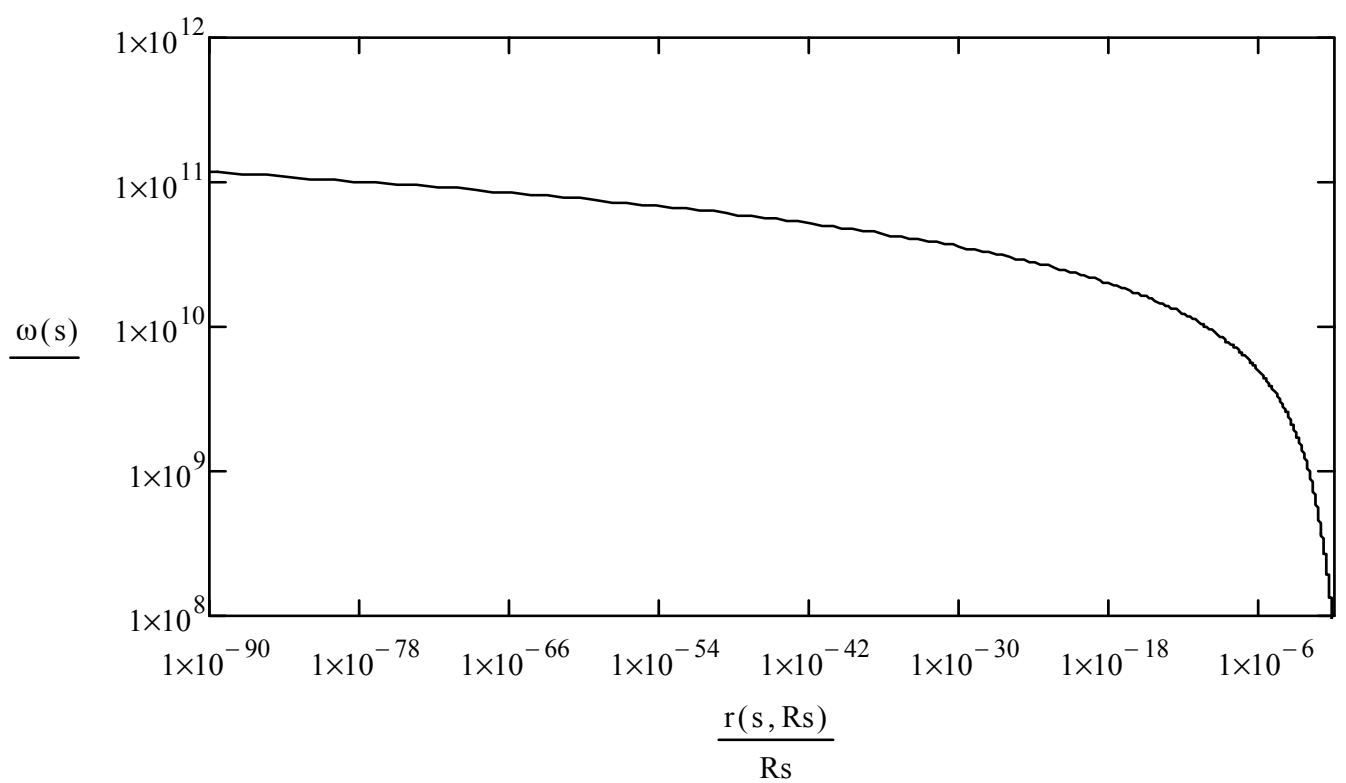

Figure 4. The dependency of the test mass orbital frequency on the natural radius normalized to $R_{s}$

\section{The Time to Impact of a Falling Test Mass in the Schwarzschild Region}

As it was derived in the previous section, the space-time in the Schwarzschild region is significantly curved. This has a profound effect on the falling time of a test mass that moves in this region. The falling time, as observed by a distant observer, is calculated from Equation 11 and Equation 12 resulting in the formula:

$$
F_{t}(\rho)=\frac{1}{c} \int_{\rho}^{\rho_{0}} \frac{e^{\frac{R_{s}}{\rho}} d \rho}{\sqrt{e^{\frac{R_{s}}{\rho}}-1}}
$$


The starting point of the fall: $\rho_{0}$, is chosen arbitrarily to be equal many times the Schwarzschild radius, on the order of thousand radii. This choice does not have a significant influence on the result of calculations. It is assumed that any test body that originates its motion at that distance was already in an equilibrium state with its environment. The fall time to a certain physical distance $\rho$, as obtained from Equation 17, is plotted in a graph shown in Figure 5. From this graph it is clear that in the Schwarzschild region of space, which has the physical radius smaller than: $\rho / R_{s}<0.01$, the fall time grows to astronomical values exceeding many times the presumed age of the universe or possibly the lifetime of any "stable" nucleus.

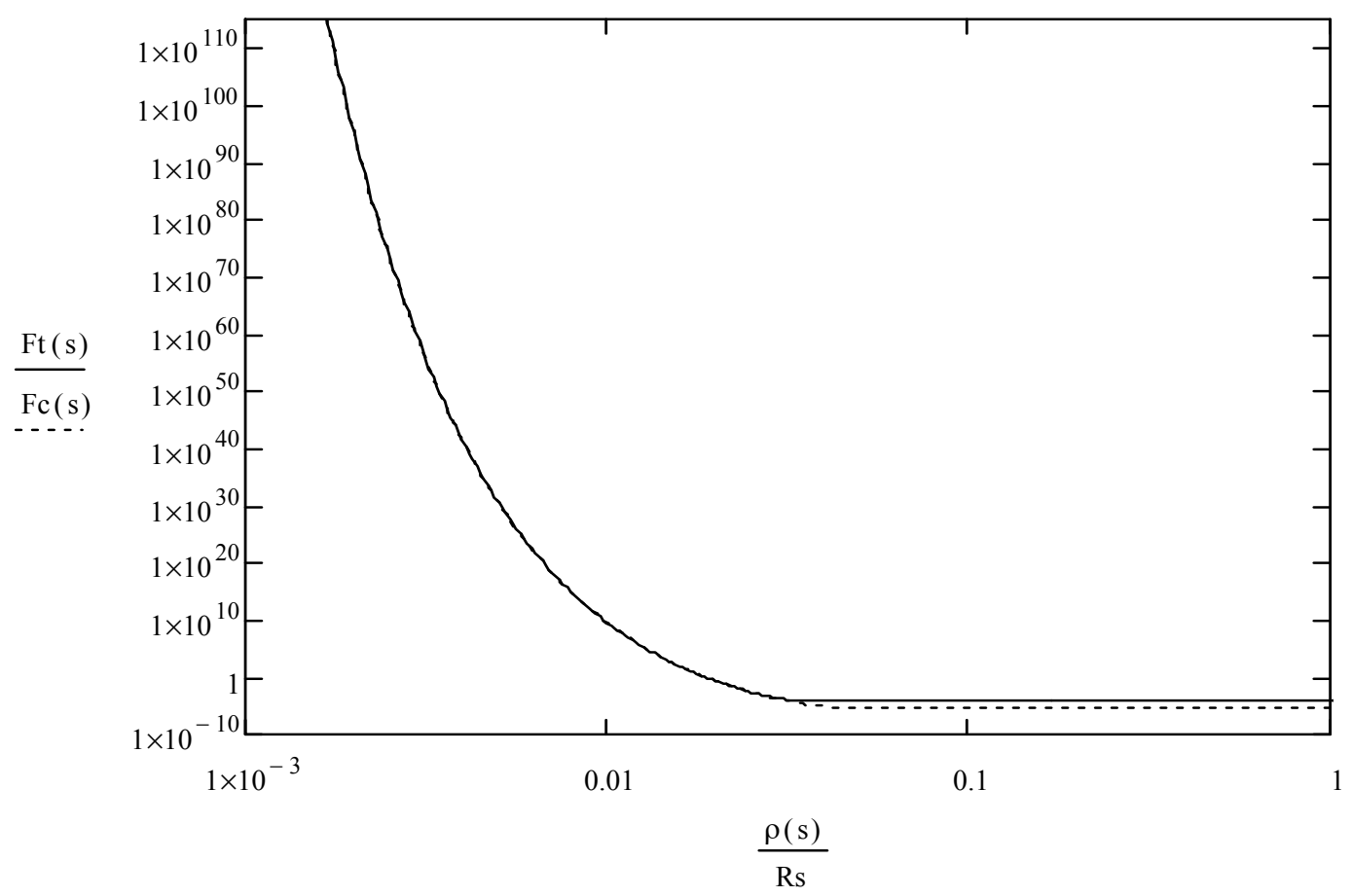

Figure 5. The fall time of a test mass from the physical distance of many times the Schwarzschild radius to the normalized physical distance of $\rho / R_{s}$. The Schwarzschild radius for this graph was set to $R_{s}=1$. The dotted line corresponds to a case when the test mass always moves with the speed close to the speed of light $c$

This graph can then be used to explain, for example, the lifetime of a neutron. As is well known neutrons consist of quarks that are their fundamental constituents. It will be therefore assumed that there is a massive central region that bounds these quarks together inside of the neutron. The quarks then give mass to this region. The electric field will be assumed compensated by the quarks and thus will be neglected. It is thus possible to visualize that the only remaining particles that can interact with this central mass are neutrinos. The size of this region is, of course, many orders of magnitude smaller than the size of the neutron, but this should be of no concern, because the neutrinos do not interact with anything else but the mass. It is thus possible to further visualize that the massive neutrino or a similar massive test body is falling down from the vicinity of the neutron to this central mass region. When this test mass collides with it, the neutron disintegrates into proton, electron, and again to a neutrino. From this graph it is then possible to find the size of this central mass region assuming that the massive impacting neutrino has a significantly smaller radius than the central region. The Schwarzschild radius that corresponds to the mass of neutron (Wikipedia, 2013) is:

$$
R_{s n}=2.487618 \cdot 10^{-54} \mathrm{~m}
$$

This is an extremely small radius, many orders of magnitude smaller than the estimated size of the neutron: $10^{-15} \mathrm{~m}$, but this should not be a problem for the calculations because the neutrino will traverse the required distance without encountering any obstructions. Using now the graph in Figure 5 or Equation 17 the time for the neutrino 
test mass impact when the central mass radius normalized to the Schwarzschild radius is set equal to: 0.003113975 , is calculated to be:

$$
F_{t}(0.003113975)=881.53 \mathrm{~s}
$$

This is a typical lifetime that is experimentally measured for a neutron (Paul, 2009). It is thus possible to conclude that the size of the central region of neutron normalized to the neutron Schwarzschild radius is equal to: 0.003113975. This is not an unreasonable value, which can indicate that there is indeed some structure there. It can also be concluded that the approximation of elementary particles as point particles is reasonable, because the natural radius that is on the order of $10^{-100} \mathrm{~m}$ is really small. Nevertheless, the new MTG metric seems to provide an interesting tool to study this region of space-time. It is thus also reasonable to consider that all elementary particles that have the central mass regions smaller are relatively stable in our cosmological timeframe and when nuclei grow in size and the corresponding central region size exceeds a certain limit, they become unstable and disintegrate.

A possible support for this hypothesis comes from the interesting measurement of nuclear decay rates and their variation in time that shows a strong correlation with the Earth-Sun distance (Jenkins et al., 2008). The Earth-Sun distance affects the Sun's gravitational potential at the Earth's orbit and this in turn affects the time of fall within the Schwarzschild region of space-time of nuclei. The reason for this effect is the gravitational mass dependency of any mass on Earth on the gravitational potential of Sun according to the relation:

$$
m_{g}=\sqrt{g_{t t}} m_{0}
$$

This formula was derived earlier by Hynecek (2005). The variation of the gravitational mass of nuclei affects their Schwarzschild radius and thus the time of fall as described by Equation 17. Another possible explanation could be related to the variations in the flow of neutrinos radiated from the Sun.

It is also reasonable to consider that all the particles in the universe will eventually disintegrate given enough time.

\section{Model of the Electron Charge}

As it was shown in the previous section, modeling of the neutron lifetime by a finite size structure located in the Schwarzschild region seems quite reasonable. This approach can thus be extended to also model charge of an electron. For example, it can be considered that there is a structure near the central region that vibrates and is producing the electromagnetic (EM) field. However, the time rate as observed from the outside is so slow that these vibrations appear as a constant electric field. The formula for the frequency as observed by a distant observer normalized to the internal proper frequency of electron is thus simply as follows:

$$
\omega_{t}(\rho)=\omega_{\tau} e^{\frac{-R_{s}}{2 \rho}}
$$

The graph of this dependency is shown in Figure 6. It is thus quite possible that the normalized frequency of EM field oscillations on the order of $10^{-150} \mathrm{~Hz}$ is perceived as a constant field appearing as generated by charge. The similar equation also holds for the radial speed of light, which follows directly from the metric in Equation 1 by setting the metric line element $d s$ to zero:

$$
c_{r}(r)=c e^{\frac{-R_{s}}{\rho(r)}}
$$

This concept would thus be consistent with the claims of Quantum Electrodynamics that photons as a propagating EM field can be viewed as an exchange force quasiparticles between electrons, because this is the same field. The author hopes that the further study of this region, including its rotation, will eventually lead to quantization and perhaps also to the resolution of mystery of the fine structure constant. This study, however, is deferred to the future.

The structures near the center of the Schwarzschild region were not specified in detail in this paper, but can be, for example, some form of strings. The metric in Equation 1 is suggesting this form, because for a small physical radius the surface area of the sphere that this metric describes is significantly contracted and the metric results in a space-time that is essentially transformed into a structure with only one time and one spatial dimension. 
Another possibility is that the central structure can be a very massive shell located near the mass equivalent physical radius. However any further discussions of details of this region at this time are just speculation, because there is no data available for the support or falsification.

The one thing is clear, however, that the electron cannot be composed of only photons or only an EM field, because it is necessary to also have a massive structure near the center of the Schwarzschild region that vibrates and provides the rest of the gravitational mass. This is consistent with the present understanding of quantum physics and what is thought the electron actually consists of.

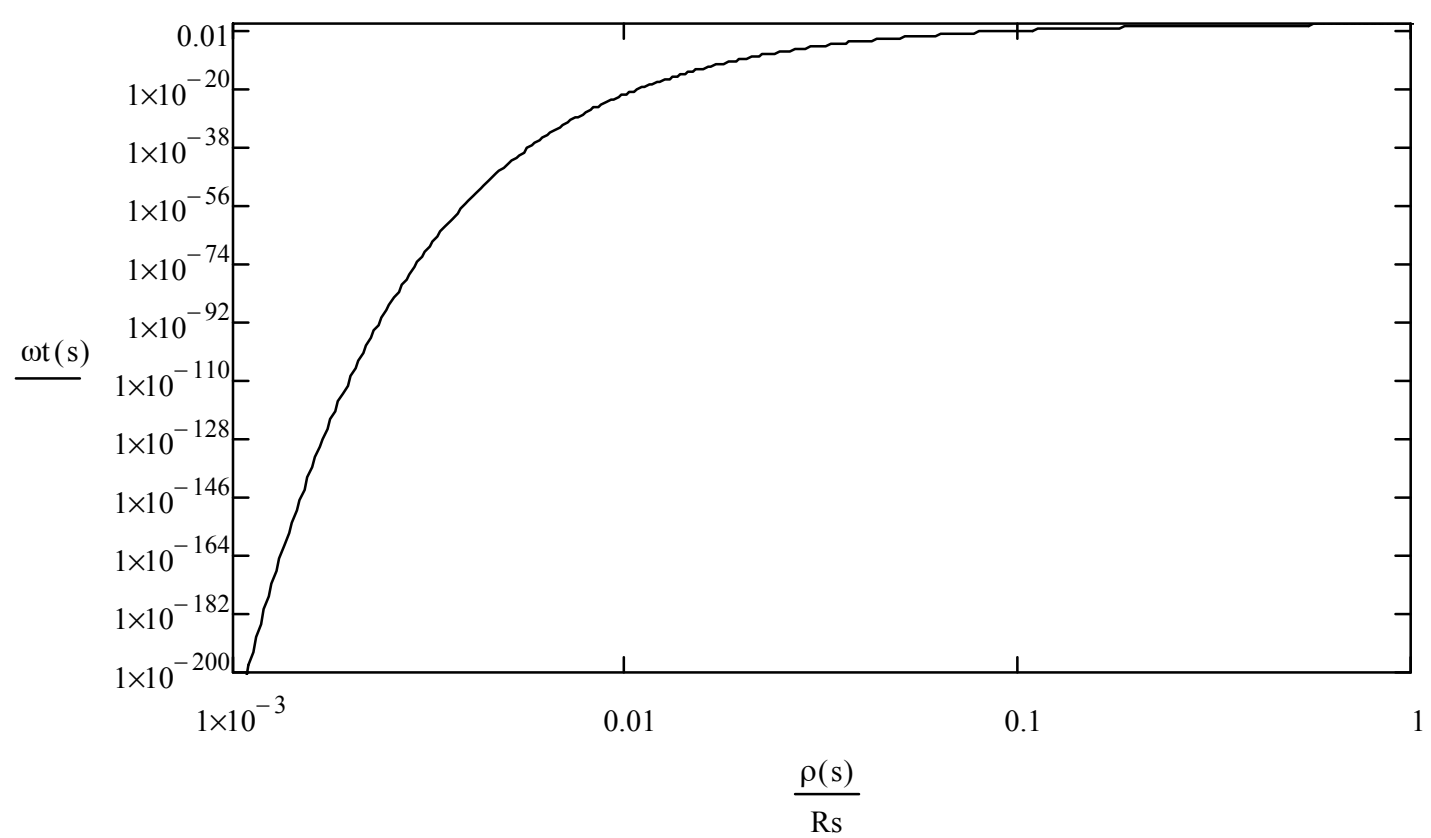

Figure 6. The graph of dependency of the frequency observed by a distant observer and normalized to the proper frequency generated by a vibrating structure within the Schwarzschild region as a function of the physical radius

\section{Conclusions}

In this paper a spherical region of space that is bounded by the Schwarzschild radius was investigated. This was possible because the metric that describes this space-time does not have the Event Horizon and the $\mathrm{BH}$ pathologies. The metric details including the Christoffel coefficients, the Riemann and Ricci tensors, and the Ricci scalar were also provided, which enabled the detail study of this region. It was found that in a certain finite region of this space-time the time, as observed by a distant observer, slows down so much that it is possible to explain charge of an electron as frozen vibrations of EM field. The same effect has also been used to explain the long neutron lifetime and the stability of other elementary nuclear particles. The experimental support for the theory was obtained from the measurement of nuclear decay rates and their correlation with the Earth-Sun distance.

\section{References}

Birrell, N. D., \& Davies, P. C. W. (1982). Quantum field in curved space, Cambridge University Press, Cambridge 1982. http://dx.doi.org/10.1017/CBO9780511622632

Hynecek, J. (2005). Remarks on the Equivalence of Inertial and Gravitational Masses and on the Accuracy ofEinstein's Theory of Gravity. Phys. Essays, 18, 182._http://dx.doi.org/10.4006/1.3025737

Hynecek, J. (2007). New Space-Time Metric, Four Tests of Gravitational Theory, and Newton's Law of Gravitation. Physics Essays Publications, 20(2), 313. http://dx.doi.org/10.4006/1.3119432

Hynecek, J. (2010). Kepler's third law for circular orbits derived in metric theory of gravity. Physics Essays publications, 23(3), 502. http://dx.doi.org/10.4006/1.3467821 
Hynecek, J. (2011). A note on the incorrect derivation of light deflection by a gravitating body in general relativity theory. Physics Essays Publications, 23(4), 588. http://dx.doi.org/10.4006/1.3495955

Hynecek, J. (2012). The Theory of Static Gravitational Field. Applied Physics Research, 4(4), 44. http://dx.doi.org/10.5539/apr.v4n4p44

Hynecek, J. (2012b). Repulsive dark matter model of the universe. Physics Essays Publications, 25(4), 561. http://dx.doi.org/10.4006/0836-1398-25.4.561

Hynecek, J. (2013). Did the Gravity Probe B Actually Prove the Einstein's General Relativity Theory? Applied Physics Research, 5(3), 90. http://dx.doi.org/10.5539/apr.v5n3p90

Jenkins, J., Fischbach, E., Buncher, J. B., Gruenwald, J. T., Krause, D. E., \& Mattes, J. (2008).Evidence for Correlations Between Nuclear Decay Rates and Earth-Sun Distance. Retrieved from http://arxiv.org/abs/0808.3283v1

NASA. (2013). http://imagine.gsfc.nasa.gov/docs/science/know_11/active_galaxies.html

Wikipedia. (2013). Retrieved from http://en.wikipedia.org/wiki/Neutron

\section{Copyrights}

Copyright for this article is retained by the author(s), with first publication rights granted to the journal.

This is an open-access article distributed under the terms and conditions of the Creative Commons Attribution license (http://creativecommons.org/licenses/by/3.0/). 